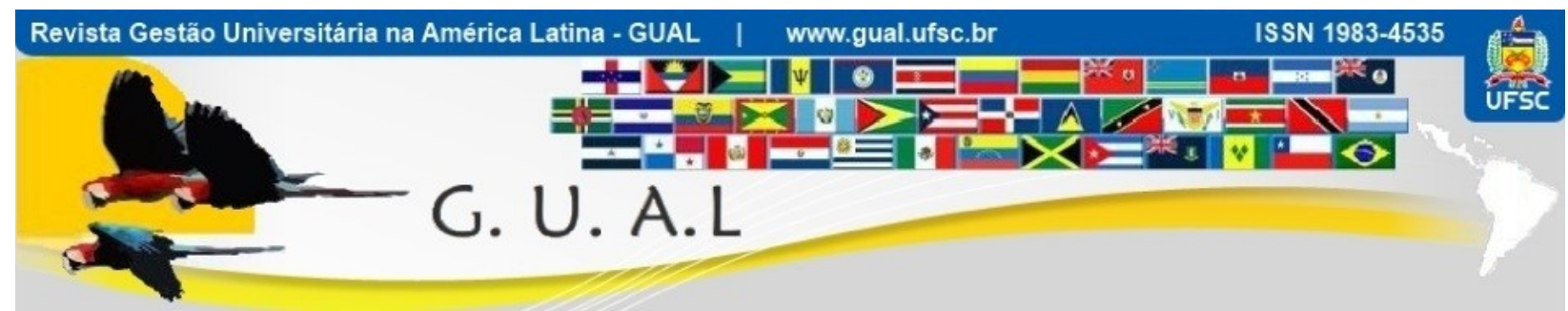

DOI: http://dx.doi.org/10.5007/1983-4535.2015v8n2p28

\title{
ASPECTOS IMPACTANTES NO DIMENSIONAMENTO DA FORÇA DE TRABALHO EM UMA INSTITUIÇÃO DE ENSINO SUPERIOR
}

\section{ASPECTS IMPACTING THE ESTIMATION OF LABOR FORCE IN A HIGHER EDUCATION INSTITUTION}

Cisne Zélia Teixeira Reis, Mestre Universidade Federal de Viçosa - UFV

cisnezelia@gmail.com

Áurea Maria Resende de Freitas, Doutoranda Universidade Federal de Viçosa - UFV aurea@ufv.br

Simone Martins, Doutoranda Universidade Federal de Viçosa - UFV simone.m@,ufv.br

Adriel Rodrigues de Oliveira, Doutor Universidade Federal de Viçosa - UFV aroli@ufv.br

Recebido em 30/setembro/2014

Aprovado em 26/março/2015

Sistema de Avaliação: Double Blind Review

Esta obra está sob uma Licença Creative Commons Atribuição-Uso. 


\title{
RESUMO
}

O dimensionamento dos recursos humanos em uma instituição merece amplo estudo, em virtude de sua complexidade. Nas Instituições Federais de Ensino Superior (IFES), com o advento da Lei $\mathrm{n}^{\circ} 11.091 / 05$, que instituiu o Plano de Carreira, este se tornou essencial e obrigatório no planejamento estratégico, como forma de identificação, análise e quantificação da força de trabalho necessária ao cumprimento dos objetivos institucionais. Numa abordagem qualitativa e quantitativa, com caráter exploratório, buscou-se investigar juntamente aos dirigentes da Universidade Federal de Viçosa as variáveis impactantes no planejamento da Gestão de Pessoas relacionadas à necessidade de ampliação ou de redução da força de trabalho. Embora os resultados apontem para a existência de eficiência na gestão de pessoas, a recomposição do quadro atual de servidores, dissociado de políticas de gerenciamento de pessoas, pode não ser uma forma eficiente para atender às demandas e ao processo de expansão das universidades federais.

Palavras-chave: Dimensionamento de Pessoal. Força de Trabalho. Gestão de Pessoas. Recursos Humanos. Universidade Federal.

\begin{abstract}
The dimensioning of workforce in an institution demands a wide study due to their complexity. In brazillian national universities, with the advent of Law No. 11091/05, the Career Plan staff has become essential and mandatory in strategic planning and institutionalized as a form of analysis and quantification of the workforce necessary for the achievement of institutional objectives. Using a qualitative and quantitative approach in an exploratory character, we tried to investigate in the Universidade Federal de Viçosa, which variables impact on the planning of people management related to the expansion or reduction of workforce.Results indicate that, in the perception of managers, there is an efficient management of people. However, the recovery of the current workers, separated by policies for people management, can not be an efficient way to meet the existing demands and the process of expansion at the federal universities.
\end{abstract}

Keywords: Dimensioning of Personnel. Labor Force. People Management. Human Resources. Federal University. 


\section{INTRODUÇÃO}

Diante de um cenário de desafios gerados no exercício da função pública, impostos pelos novos papéis do Estado, as Instituições Federais de Ensino brasileiras vivenciam a necessidade de estruturação para se adaptarem à nova ordem pública. Desde 1991, com o advento da Lei 8.112/90 (BRASIL, 1990), que trata do regime jurídico dos servidores públicos civis da União, das autarquias e das fundações públicas federais, verifica-se significativa redução no quantitativo de pessoal, sem a correspondente recomposição.

Em 2005, com a Lei no 11.091 (BRASIL, 2005), que instituiu o Plano de Carreira dos Cargos Técnico-Administrativos em Educação, no âmbito das Instituições Federais de Ensino vinculadas ao Ministério da Educação, foi estabelecido que o Plano de Desenvolvimento dos Integrantes do Plano de Carreira deverá conter o dimensionamento das necessidades institucionais, com definição de modelos de alocação de vagas que contemplem a diversidade da instituição.

Assim, avaliar as políticas de dimensionamento dos recursos humanos em uma instituição tem sua importância em face de sua complexidade. Dentre as diversas funções de gestão de pessoas, o planejamento passa a ocupar papel estratégico.

Proceder ao planejamento da força de trabalho requer um processo sistemático e contínuo de avaliação das necessidades atuais e futuras de recursos humanos, relacionada ao quantitativo, composição e perfil, e de definição das estratégias e ações que se fazem necessárias para viabilizar o alcance de tais necessidades.

Levando-se em conta a importância da força de trabalho na prestação de serviços de qualidade, o seu dimensionamento é, portanto, essencial na gestão de pessoas. Entretanto, trata-se de trabalho complexo, composto por variáveis diversas, que precisa percorrer etapas para sua realização, sendo necessário, a priori, um diagnóstico em que sejam levados em consideração, inclusive, aspectos relacionados à compatibilidade das habilidades dos servidores com as funções desempenhadas e à habilidade gerencial dos dirigentes.

Atualmente, as Instituições Federais de Ensino passam por um momento de grande expansão, em face da implementação de políticas do Ministério da Educação para ampliar o acesso e a permanência na educação superior. As políticas de aumento de vagas, ampliação ou abertura de cursos noturnos, aumento do número de alunos por professor, redução do custo por aluno, flexibilização de currículos e combate à evasão provocam impactos na área de 
gestão de pessoas, demandando esforços contínuos no desenvolvimento e implementação de estratégias para suprir as demandas pactuadas.

Considerando o exposto, uma questão é levantada: Para atender às demandas e ao processo de expansão em curso é necessário ampliar o quadro de pessoal ou trabalhar os aspectos relacionados ao gerenciamento de pessoas?

Diante disso, buscou-se traçar um diagnóstico fundamentado nos dados apresentados na literatura e na percepção dos dirigentes da Universidade Federal de Viçosa, acerca da necessidade de ampliação do quadro de pessoal técnico-administrativo para atendimento das demandas previstas no planejamento de curto prazo e daquelas impostas pela expansão. Buscou-se, também, diagnosticar a percepção dos dirigentes sobre as variáveis consideradas impactantes ou de relevância para definição de quadro de pessoal, tendo como parâmetro seu planejamento e os processos de trabalho.

Assim, a relevância do estudo está em fornecer informações complementares para dimensionar a necessidade de pessoal, permitir aproximar os gestores da realidade institucional, conhecer prováveis deficiências ou méritos na gestão de pessoas descentralizada, além de contribuir para proposição de políticas que visem à valorização e satisfação do servidor.

\section{REVISÃO BIBLIOGRÁFICA}

\subsection{GESTÃO DE PESSOAS}

A Administração de Recursos Humanos no Brasil, segundo França (2007), vivenciou seis fases evolutivas: antes de 1930, o período pré-juridico trabalhista, caracterizado pela inexistência de legislação trabalhista; de 1930 a 1950, o período burocrático, caracterizado pelo advento da legislação trabalhista e surgimento de departamento de pessoal; de 1950 a 1960, o período tecnicista, caracterizado pelo surgimento dos Sistemas de Recursos Humanos; de 1960 a 1980, o período da abordagem sistêmica, caracterizado pelo surgimento dos primeiros cargos de gerência de recursos humanos e práticas de gestão participativa e de qualidade; de 1980 a 1990, o período de relações industriais integradas, caracterizado pela integração dos enfoques administrativos, estruturalista e comportamental; e, a partir de 1990, o período caracterizado pelas reformas estruturais profundas sob os desafios de qualidade e competitividade, tornando-se necessária a administração estratégica. 


\section{ASPECTOS IMPACTANTES NO DIMENSIONAMENTO DA FORÇA DE TRABALHO EM UMA \\ INSTITUIÇÃO DE ENSINO SUPERIOR \\ DOI: http://dx.doi.org/10.5007/1983-4535.2015v8n2p28}

A abordagem estratégica de gestão de pessoas envolve o entendimento das pessoas como recurso para a obtenção de vantagem competitiva; uso de planejamento; coerência entre políticas, práticas de emprego e a estratégia de negócio; e tomada de decisão sobre os aspectos da relação de emprego no mais alto nível hierárquico (SISSON; STOREY, 2000 apud LACOMBE; CHU, 2008).

O cenário de gestão de pessoas com ações corporativas estratégicas resulta na sintonia entre as ações e as metas organizacionais. Nesta perspectiva, argumenta França (2007), o modelo de gestão de recursos humanos tem abordagem focada em elementos como valorização do talento humano, atração e manutenção de pessoas, motivação e mobilidade, diagnóstico, gestão de informação e políticas integradas.

Para Dessler (2003), o fato de as pessoas serem, hoje, fundamental na busca de vantagem competitiva coloca o RH em um papel central e envolvido no desenvolvimento e na implementação de estratégias, possibilitando o fortalecimento da competitividade organizacional e formação de equipes de trabalho.

No Setor Público, a administração de recursos humanos possui peculiaridades que são oriundas da própria natureza das organizações que compõem esse setor. A organização do setor privado diverge quanto a finalidade, os meios utilizados para recrutamento, seleção, contratação, as políticas de remuneração, os métodos de avaliação de desempenho, além de outros. Dessa forma, quando se refere a recursos humanos no setor público, é necessário atentar para uma realidade diversa daquela já consagrada nas organizações privadas (FERREIRA et al. 2008).

Independentemente do setor, a efetividade da gestão estratégica de pessoas, de acordo com Dutra (2002), está ligada à clareza, ao objetivo da empresa em relação às pessoas. Esta clareza permitirá maior efetividade nos seguintes aspectos, dentre outros: planejamento e dimensionamento do quadro e a definição das necessidades e das políticas de movimentação de pessoas.

\subsection{PLANEJAMENTO DA FORÇA DE TRABALHO}

O planejamento de recursos humanos, conforme Lucena (1991), compreende o processo gerencial de identificação e análise das necessidades organizacionais e o consequente desenvolvimento de políticas, programas, sistemas e atividades que satisfaçam essas necessidades, a curto, médio e longo prazos, tendo em vista assegurar a realização das 


\section{ASPECTOS IMPACTANTES NO DIMENSIONAMENTO DA FORÇA DE TRABALHO EM UMA \\ INSTITUIÇÃO DE ENSINO SUPERIOR \\ DOI: http://dx.doi.org/10.5007/1983-4535.2015v8n2p28}

estratégias do negócio, dos objetivos da empresa e de sua continuidade sob condições de mudanças.

É essencial, portanto, o planejamento do quadro de pessoal, para que se possa determinar lacunas e excessos no presente e no futuro, permitindo, assim, que a empresa tome decisões em relação às pessoas e que estas possam orientar-se em seu desenvolvimento e carreira. A captação de pessoas deve ser realizada com o conhecimento de necessidades futuras ou excessos presentes, para garantir o seu alinhamento com as estratégias da empresa e permitir melhor utilização dos recursos disponíveis, tanto interna quanto externamente (DUTRA, 2002).

Para o planejamento da força de trabalho, conforme Dessler (2003), é necessário que no processo de formulação do plano para preenchimento das vagas futuras seja levada em consideração a projeção das posições que devem se abrir e das que serão preenchidas tanto na área operacional, técnica e estratégica da empresa.

O plano estratégico será o ponto de partida para as políticas de recursos humanos, na formação da equipe, condução e aperfeiçoamento. Ao planejar a equipe, deve-se basear no plano estratégico da empresa, caso contrário as equipes poderão ser superdimensionadas ou formadas aquém do necessário em quantidade e qualidade. $\mathrm{O}$ plano estratégico irá indicar quais as pessoas necessárias, suas qualificações e o que elas precisam fazer para atingir os objetivos previstos no planejamento. Não existe uma equipe ideal para todas as situações, uma vez que cada empresa tem suas peculiaridades, seu ambiente, sua cultura, seus pontos fortes e fracos, suas ameaças e oportunidades, seus processos, seus produtos, dentre outros. A formação da equipe é um processo contínuo, em razão das constantes mudanças nos planos da empresa, o que resulta em alterações nas suas necessidades. Além disso, as pessoas mudam: envelhecem, aumentam sua experiência, ganham ou perdem motivação, dentre outros fatores e, como consequência, é preciso constantemente avaliar e planejar a força de trabalho (LACOMBE, 2005).

Para Lucena (1991), o planejamento de recursos humanos deve abranger a previsão de necessidades de recursos humanos requeridas pelo negócio da empresa - essa informação possibilita dimensionar e formular decisões e projetos de trabalho; a análise dos recursos humanos em relação às metas estratégicas - objetiva analisar quantitativa e qualitativamente o quanto a organização dispõe de pessoal para suprir suas necessidades, a curto, médio e longo prazos, bem como identificar a carência de capacitação e as necessidades de política de gestão 


\section{ASPECTOS IMPACTANTES NO DIMENSIONAMENTO DA FORÇA DE TRABALHO EM UMA \\ INSTITUIÇÃO DE ENSINO SUPERIOR \\ DOI: http://dx.doi.org/10.5007/1983-4535.2015v8n2p28}

de recursos humanos; e a análise do ambiente e do mercado de trabalho - que possibilita apontar indicadores para equacionar as necessidades da empresa.

De acordo com Dutra (2002), as organizações que obtiveram sucesso com o planejamento do quadro de pessoas estabeleceram as seguintes práticas: desvincularam o planejamento do quadro de pessoas do desenho organizacional da empresa; vincularam o planejamento do quadro de pessoas aos processos essenciais da empresa; consideraram o aumento da complexidade tecnológica da atividade da empresa; e avaliaram a capacidade das pessoas atenderem às necessidades do presente e do futuro da empresa.

Para Nogueira (2005), a efetividade da ação estatal depende inteiramente de um planejamento cuidadoso e de longo prazo da força de trabalho do setor público, que tenha um lugar destacado entre as políticas públicas.

\subsection{DIMENSIONAMENTO DA FORÇA DE TRABALHO}

O dimensionamento do quadro, segundo Dutra (2002), é essencial para que a empresa possa preparar-se e orientar as pessoas. No dimensionamento da força de trabalho, a situação atual do quadro deve ser considerada conjuntamente com a aquisição de tecnologia, a reestruturação do processo produtivo, a estruturação de novos modelos de gestão, bem como a qualificação e o desenvolvimento das pessoas do quadro e as contratações/demissões, tudo isso confrontado com o formato da situação planejada para o quadro. Essa verificação permitirá conhecer as lacunas e sobras em relação aos diferentes níveis de carreira ou diferentes níveis de complexidade dos processos essenciais.

Dimensionar e planejar as necessidades de recursos humanos a longo/médio/curto prazos para atender às exigências e aos objetivos da empresa exige busca de técnicas apropriadas à realidade de cada empresa. Os pressupostos básicos do planejamento de recursos humanos irão definir as diretrizes básicas e a decisão sobre a viabilidade do planejamento, que, segundo Lucena (1991), se não se caracterizar como utopia, com certeza representará um grande desafio.

Uma política de aplicação de recursos humanos deve contemplar critérios de planejamento, alocação e movimentação interna de recursos humanos, bem como determinar a quantidade de recursos humanos necessária e a alocação desses recursos em termos de posicionamento dentro da organização (CHIAVENATO, 2006). 


\section{ASPECTOS IMPACTANTES NO DIMENSIONAMENTO DA FORÇA DE TRABALHO EM UMA \\ INSTITUIÇÃO DE ENSINO SUPERIOR \\ DOI: http://dx.doi.org/10.5007/1983-4535.2015v8n2p28}

Para o novo perfil da força de trabalho que está se delineando, segundo Pacheco (2002), tanto contribuíram as precoces e vantajosas aposentadorias dos "apreensivos", como a política de concursos anuais direcionados para cargos de nível superior. Mais relevante do que as causas, parecem ter sido os resultados — de que a força de trabalho vai assumindo um perfil compatível com os desafios à função pública impostos pelos novos papéis do Estado, e consistente com as propostas para a reforma do aparelho do Estado. As medidas adotadas tentam conciliar a restrição fiscal com a condução da mudança do perfil e da qualificação dos recursos humanos. Dimensionar a força de trabalho do setor público traz à tona questões profundas como a natureza do federalismo brasileiro, a relação entre poderes, as desigualdades regionais, as injustiças sociais e os privilégios, a bandeira fácil da isonomia que, por trás da aparência de "tratamento igual a todos", esconde a falta de coragem para avaliar e recompensar os desempenhos diferentes; as dificuldades em entender e aceitar o sentido profundo da meritocracia, etc.

O objetivo de uma política de recursos humanos no setor público, via de regra, é alcançar o perfil desejado para a força de trabalho e sua atuação de forma motivada e eficiente, em um modelo de gestão que vise o alcance de resultados e o atendimento satisfatórios ao cidadão em um cenário de restrição orçamentária (MARCONI, 2004).

Os trabalhos de dimensionamento do quadro de pessoal publicados têm evidenciado uma evolução, principalmente na implementação de modelos para a área de saúde, contemplando grande número de variáveis, permitindo melhor visibilidade da realidade, e gerando maior complexidade para sua operacionalização (GAIDZINSKI, 1998).

No âmbito das Instituições Federais de Ensino, vinculadas ao Ministério da Educação, o dimensionamento das necessidades institucionais, com definição de modelos de alocação de vagas que contemplem a diversidade da instituição, ocupa espaço no planejamento e política de recursos humanos, principalmente em função da Lei $n^{0}$ 11.091/05 (BRASIL, 2005), que institui o Plano de Carreira dos Cargos Técnico-Administrativos em Educação e o Decreto n ${ }^{\circ}$ 5.825/06 (BRASIL, 2006), que estabelece as diretrizes para elaboração do Plano de Desenvolvimento dos Integrantes do Plano de Carreira dos Cargos Técnico-Administrativos em Educação. Apesar da importância para o planejamento institucional e da exigência legal, não foi possível identificar casos na literatura que permitissem uma avaliação, o que justifica o presente estudo. 


\section{PROCEDIMENTOS METODOLÓGICOS}

Este trabalho visa aferir as variáveis que podem interferir no processo de dimensionamento da força de trabalho, relacionadas com o gerenciamento de recursos humanos em uma organização pública de ensino, para identificar se há necessidade de ampliação da força de trabalho.

Optou-se por fazer um estudo de caso numa universidade federal, visto que, por imposições legais, estas instituições foram obrigadas a implementar estudos sobre o dimensionamento de sua força de trabalho, para posteriormente propor metodologia de alocação de vagas, considerando o processo expansionista em curso nas universidades federais brasileiras. De acordo com Yin (2002, p. 32) "um estudo de caso é uma investigação empírica que investiga um fenômeno contemporâneo dentro de seu contexto da vida real, especialmente quando os limites entre o fenômeno e o contexto não estão claramente definidos".

O estudo foi realizado nos meses de agosto e setembro de 2008, na Universidade Federal de Viçosa (UFV), Viçosa-MG, localizada na Zona da Mata mineira, que, na época, possuía 939 docentes, 2.365 técnicos administrativos e 15.021 alunos de graduação, pósgraduação e nível médio.

Para a classificação da pesquisa considerou-se o critério adotado por Vergara (2006), que a qualifica em relação a dois aspectos: quanto aos fins e quanto aos meios. A pesquisa caracteriza-se quanto aos fins como exploratória e descritiva. Exploratória porque foi realizada em uma área com pouco conhecimento acumulado. Descritiva porque expõe características de determinada população ou de determinado fenômeno. Quanto aos meios, a pesquisa foi bibliográfica, documental, de campo e estudo de caso.

A coleta de dados primários foi realizada por meio da aplicação de questionário eletrônico estruturado, composto por 28 questões, cujo objetivo foi identificar a necessidade de ampliação da força de trabalho da UFV e, ainda, aferir a percepção dos dirigentes a respeito das variáveis relacionadas ao gerenciamento de recursos humanos. Para identificação das variáveis para composição do questionário formou-se um grupo focal com os membros da comissão responsável por proceder aos estudos de dimensionamento das necessidades institucionais de pessoal técnico-administrativo. Morgan (1997) define grupos focais como técnica de pesquisa que coleta dados por meio das interações grupais ao se discutir um tópico especial. 


\section{ASPECTOS IMPACTANTES NO DIMENSIONAMENTO DA FORÇA DE TRABALHO EM UMA INSTITUIÇÃO DE ENSINO SUPERIOR \\ DOI: http://dx.doi.org/10.5007/1983-4535.2015v8n2p28}

Assim, o questionário foi dividido em oito partes, sendo inseridas questões para identificar: 1) Necessidade de ampliação da força de trabalho; 2) Possibilidade de redução da força de trabalho; 3) Capacitação; 4) Gestão de pessoal; 5) Idade; 6) Motivação; 7) Saúde Ocupacional; 8) Volume de trabalho. As questões foram associadas a uma escala categórica sendo as opiniões nominalmente mensuradas em quatro categorias de respostas: N=Nunca; $\mathrm{E}=$ Eventualmente; $\mathrm{F}=$ Frequentemente; e $\mathrm{S}=$ Sempre. Foi utilizado o programa SPSS versão 15.0, para análise de frequência das questões objetivas dos questionários.

Os dados secundários, como informações cadastrais dos servidores e relatórios gerenciais, foram coletados junto ao órgão responsável pela Gestão de Pessoas da UFV, com o propósito de subsidiar a análise dos dados primários, sendo tratados de forma qualitativa, por meio de análise descritiva e estruturada.

Participaram da pesquisa dirigentes da UFV, ocupantes dos cargos de Reitor, PróReitores, Diretores de Centro e de unidades administrativas e Chefes de unidades acadêmicas e administrativas, totalizando 79 indivíduos pesquisados, dos quais se obteve $84 \%$ de respostas.

\section{ANÁLISE DOS RESULTADOS}

No ano de 2008, seguindo instruções constantes da Lei no 11.091/05 (BRASIL, 2005) e do Decreto $n^{\circ}$ 5.825/06 (BRASIL, 2006), a Universidade Federal de Viçosa (UFV) deu início às atividades referentes ao Dimensionamento da Força de Trabalho do Pessoal TécnicoAdministrativo, objetivando elaborar um diagnóstico, considerando os aspectos qualitativos e quantitativos, que, posteriormente, subsidiará a elaboração do Modelo Interno de Alocação de Pessoal Técnico-Administrativo.

Para isso, desenvolveu-se um sistema computacional para coleta de dados, no qual os dirigentes prestaram as informações referentes a cargos e lotação real do servidor; atividades desenvolvidas pela Unidade e suas Subunidades; a força de trabalho efetiva e não efetiva; justificativas para ampliação do quadro de pessoal; e, ainda, questionário on-line para permitir identificar variáveis relacionadas ao gerenciamento de recursos humanos, sendo este último o objeto de estudo deste artigo.

Ao apurar a opinião dos dirigentes sobre as questões básicas e genéricas: 1) "O atual quadro de pessoal atende ao planejamento de curto prazo?" e 2) "O atual quadro de pessoal atende à necessidade/previsão de expansão?", verificou-se que para 77,3\% deles, conforme 
Figura 1, o atual quadro de servidores não é suficiente para o cumprimento das atividades previstas no planejamento de curto prazo. Situação que se torna agravante quando se trata de necessidade de pessoal para o atendimento às demandas impostas pela expansão institucional, o que pode ser visto também na Figura 1, em que 92,4\% dos dirigentes apontam o atual quadro de pessoal como insuficiente.

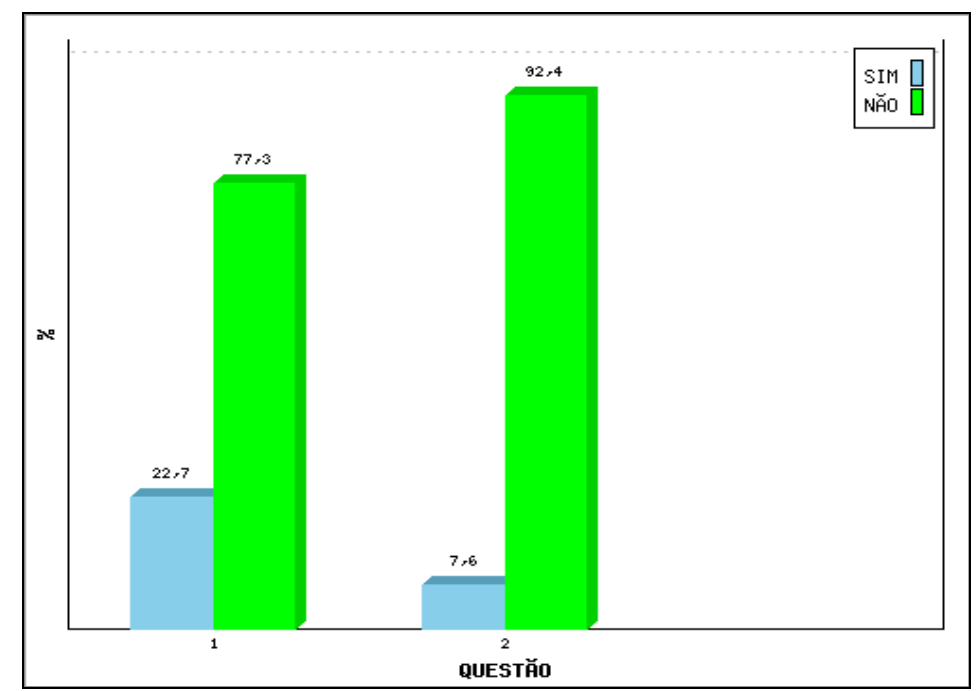

Figura 1 Resultado da consulta global para UFV nas questões 1 e 2. Fonte: Dados da pesquisa.

Corroborando esta manifestação sobre a necessidade de ampliação da força de trabalho, observa-se na Tabela 1 que, no período de 1996 a 2003, o número de servidores públicos federais civis ativos do poder executivo, incluindo o Ministério da Educação, reduziu. Somente a partir do ano 2004 esta força de trabalho vem sendo gradativamente reconstituída. No caso da Instituição em estudo verifica-se que não houve reconstituição do seu quadro de pessoal, uma vez que o número de aposentadorias ocorridas é superior às autorizações de provimento no quadro de pessoal docentes e técnicos de nível superior e médio. 


\section{ASPECTOS IMPACTANTES NO DIMENSIONAMENTO DA FORÇA DE TRABALHO EM UMA \\ INSTITUIÇÃO DE ENSINO SUPERIOR \\ DOI: http://dx.doi.org/10.5007/1983-4535.2015v8n2p28}

Tabela 1 Quantitativo dos Servidores Públicos Federais Civis Ativos

\begin{tabular}{|cccc|}
\hline ANO & $\begin{array}{c}\text { Universidade } \\
\text { Federal de } \\
\text { Viçosa* }\end{array}$ & $\begin{array}{c}\text { Ministério da } \\
\text { Educação }\end{array}$ & Poder Executivo \\
\hline 1996 & 3.762 & 179.643 & 554.736 \\
1997 & 3.675 & 174.966 & 531.725 \\
1998 & 3.584 & 168.403 & 513.720 \\
1999 & 3.532 & 165.510 & 497.125 \\
2000 & 3.492 & 165.595 & 486.912 \\
2001 & 3.411 & 163.479 & 485.303 \\
2002 & 3.446 & 165.163 & 485.741 \\
2003 & 3.360 & 164.870 & 485.980 \\
2004 & 3.388 & 171.925 & 499.138 \\
2005 & 3.361 & 173.181 & 508.963 \\
2006 & 3.319 & 179.449 & 528.124 \\
2007 & 3.276 & 180.895 & 528.420 \\
2008 & 3.264 & 188.004 & 538.797 \\
\hline
\end{tabular}

*Total de servidores técnico-administrativos e docentes

Fonte: MP-Boletim Estatístico de Pessoal, nº 152, Dez.2008 (BRASIL, 2008) e Relatório de Atividades da UFV (Sabioni et al., 2004, 2008, 2009)

Os resultados demonstram efetiva redução do quadro de pessoal, em detrimento de políticas governamentais de recomposição. Entretanto, apenas a recomposição do quadro, dissociado de políticas de gerenciamento de pessoas, pode não ser uma forma eficiente para atender às demandas e ao processo de expansão. Acredita-se que fatores relativos ao gerenciamento dos recursos humanos devem ser considerados para que se possa identificar se o aumento do quantitativo é a solução ou se há necessidade de adequação das variáveis relacionadas ao gerenciamento de pessoal.

Analisando a percepção dos dirigentes quanto às variáveis do questionário, verificouse que:

\subsection{NECESSIDADE DE AMPLIAÇÃO DA FORÇA DE TRABALHO}

Os dirigentes, em sua maioria, manifestarem-se positivamente quando questionados a respeito da necessidade de ampliação da força de trabalho, indicando haver carência de pessoal. Isto, porque, conforme Tabela 2, para 34,85\% e 28,79\% dos respondentes, frequentemente e sempre, respectivamente, observa-se a ocorrência de sobrecarga de trabalhos, apesar de contarem com o apoio de bolsistas ou estagiários e, ainda, terceirizados para o desempenho das tarefas rotineiras, exercendo atividades em caráter permanente.

Em razão dos impedimentos legais para contratação de pessoal efetivo, principalmente para as atividades de manutenção, conservação e segurança, a Instituição ampliou muito a 
contratação de serviços terceirizados. Conforme dados constantes no Relatório de Gestão de 2008 (UFV, 2009), verificou-se crescimento de 371\% nesse tipo de contratação, no período de 2003 a 2008.

Tabela 2 Questões da Variável Ampliação

\begin{tabular}{|lcccc}
\hline & \multicolumn{3}{c}{$\mathbf{\%}$} \\
\cline { 2 - 5 } & \multicolumn{1}{c}{$\mathbf{N}$} & $\mathbf{E}$ & $\mathbf{F}$ & $\mathbf{S}$ \\
\hline $\begin{array}{l}\text { Há sobrecarga de trabalho para os servidores. } \\
\text { Os funcionários terceirizados exercem atividades de caráter } \\
\text { permanente. }\end{array}$ & 1,52 & 34,85 & 34,85 & 28,79 \\
$\begin{array}{l}\text { Os estagiários ou bolsistas exercem atividades de caráter } \\
\text { permanente. }\end{array}$ & 18,18 & 3,03 & 10,61 & 68,18 \\
\hline \multicolumn{1}{c}{ Total } & 16,67 & 16,67 & 25,76 & 40,91 \\
\hline
\end{tabular}

$\mathrm{N}=$ Nunca; $\mathrm{E}=$ Eventualmente; $\mathrm{F}=$ Frequentemente; e $\mathrm{S}=$ Sempre.

Fonte: Dados da pesquisa.

\subsection{POSSIBILIDADE DE REDUÇÃO DA FORÇA DE TRABALHO}

Para Dutra (2002), é importante considerar a necessidade de ampliação ou redução do quadro de pessoal, pois a confrontação da situação atual com a desejada permite verificar as lacunas e as sobras em relação ao quadro e à complexidade dos processos essenciais. Para suprir às lacunas, a organização poderá desenvolver as pessoas internamente ou contratar efetivos ou prestadores de serviço. E, no caso de excesso de contingente, deve-se avaliar a transferência das pessoas ou o desligamento dessas.

Se por um lado as instituições de ensino encontram-se em meio ao processo expansionista, por outro, há na Instituição um esforço para modernização dos processos. Assim foram inseridas questões para identificar a possibilidade de redução de pessoal em virtude da inovação tecnológica ou da escassez de recursos.

Observou-se, portanto, que, conforme Tabela 3, para a maioria dos respondentes (77,28\%), nunca ou eventualmente, a necessidade de pessoal se reduziria em virtude da modernização dos processos e inovação tecnológica, fato que traria benefícios para a realização dos trabalhos, contudo não descarta a necessidade de pessoal e não resulta em ociosidade funcional no serviço. Além disso, verifica-se que mesmo em caso de ocorrência de restrições orçamentárias, situação em que, a priori, o nível de atividades reduziria, a necessidade de pessoal para atender às demandas se mantém. 


\section{ASPECTOS IMPACTANTES NO DIMENSIONAMENTO DA FORÇA DE TRABALHO EM UMA \\ INSTITUIÇÃO DE ENSINO SUPERIOR \\ DOI: http://dx.doi.org/10.5007/1983-4535.2015v8n2p28}

Tabela 3 Questões da Variável Redução

\begin{tabular}{|lcccc|}
\hline \multicolumn{1}{c}{ ITEM } & \multicolumn{3}{c}{$\mathbf{\%}$} \\
\cline { 2 - 5 } & $\mathbf{N}$ & $\mathbf{E}$ & $\mathbf{F}$ & $\mathbf{S}$ \\
\hline A inovação tecnológica reduz a necessidade de pessoal. & 22,73 & 54,55 & 13,64 & 9,09 \\
A modernização dos processos de trabalho reduz a & & & & 7,58 \\
necessidade de pessoal. & 21,21 & 57,58 & 13,64 & \\
A escassez de recursos materiais e financeiros induz à & & & 7,03 \\
ociosidade do pessoal. & 50,00 & 39,39 & 7,58 & - \\
\hline \multicolumn{1}{r}{ Há ociosidade funcional no serviço. } & 51,52 & 43,94 & 4,55 & $\mathbf{4 , 9 2}$ \\
\hline
\end{tabular}

$\mathrm{N}=$ Nunca; $\mathrm{E}=$ Eventualmente; $\mathrm{F}=$ Frequentemente; e $\mathrm{S}=$ Sempre.

Fonte: Dados da pesquisa.

Estas respostas também guardam correlação com as questões básicas e genéricas feitas inicialmente, em que se constada a insuficiência de pessoal para atender ao planejamento de curto prazo ou à expansão.

\subsection{CAPACITAÇÃO}

Considerando que a capacitação é essencial para melhorar a qualidade dos serviços prestados e atender maior demanda, introduziram-se questões para identificar na percepção dos dirigentes quanto ao nível de capacitação de seu quadro de pessoal.

Como resultado, verifica-se, na Tabela 4, que nenhum dirigente considerou que o pessoal sob sua supervisão seja despreparado para o trabalho. Além disso, nota-se que os dirigentes, na grande maioria, aproximadamente $97 \%$, são de opinião de que, frequentemente e sempre, sua equipe de trabalho está capacitada para a realização de trabalho com a devida qualidade, muito embora a Política de Capacitação de servidores na Instituição seja recente e não esteja ainda amplamente implantada com profissionais de diversas áreas, ou seja, carecendo ainda de capacitação.

De acordo com dados do órgão responsável pela Gestão de Pessoas, 31,08\% dos servidores não receberam nenhum curso de capacitação no período compreendido entre 2005 e 2008 e $37 \%$ destes não possuem ensino médio. 


\section{ASPECTOS IMPACTANTES NO DIMENSIONAMENTO DA FORÇA DE TRABALHO EM UMA \\ INSTITUIÇÃO DE ENSINO SUPERIOR \\ DOI: http://dx.doi.org/10.5007/1983-4535.2015v8n2p28}

Tabela 4 Questões da Variável Capacitação

\begin{tabular}{lcccc}
\hline \multicolumn{1}{c}{ ITEM } & \multicolumn{3}{c}{$\mathbf{\%}$} \\
\cline { 2 - 5 } & $\mathbf{N}$ & $\mathbf{E}$ & $\mathbf{F}$ & $\mathbf{S}$ \\
\hline O pessoal demonstra ter habilidade para exercer suas funções. & - & 3,03 & 59,09 & 37,88 \\
O pessoal possui capacidade técnica para desempenhar suas & & & & \\
atividades. & - & 3,03 & 62,12 & 34,85 \\
O pessoal desempenha os trabalhos a contento. & - & 7,58 & 65,15 & 27,27 \\
A qualidade do trabalho atende às exigências da Unidade. & - & 10,61 & 59,09 & 30,30 \\
\hline \multicolumn{1}{c}{ Total } & - & $\mathbf{6 , 0 6}$ & $\mathbf{6 1 , 3 6}$ & $\mathbf{3 2 , 5 8}$ \\
\hline
\end{tabular}

$\mathrm{N}=$ Nunca; $\mathrm{E}=$ Eventualmente $\mathrm{F}=$ Frequentemente; e $\mathrm{S}=$ Sempre

Fonte: Dados da pesquisa.

A avaliação do nível de capacitação do quadro, segundo Dutra (2002), é um fator importante no dimensionamento do quadro de pessoal, devendo em sua análise considerar a capacidade das pessoas em relação ao grau de preparo em que se encontram para assumir as atividades de maior complexidade nos processos essenciais da organização, bem como atender às necessidades do presente e do futuro da empresa.

Com os resultados foi possível inferir que, possivelmente, na UFV há uma política de empregabilidade, ou seja, os servidores são responsáveis por suas carreiras e pela sua capacitação, ou que a capacitação ocorre por meio da prática funcional nesta Instituição.

\subsection{GESTÃO DE PESSOAL}

Como resultado, verifica-se, na Tabela 5, que em média mais de $70 \%$ das respostas apontam uma supervisão eficiente em termos de adequação da jornada de trabalho e da otimização da mão de obra. Entretanto, um percentual muito alto, 66,67\%, considera que ocorra o retrabalho, podendo ser indício de falta de capacitação e, ou, de uso de mais pessoas para a realização de um mesmo trabalho, o que pode implicar necessidade de pessoal que não condiz com a realidade. 


\section{ASPECTOS IMPACTANTES NO DIMENSIONAMENTO DA FORÇA DE TRABALHO EM UMA \\ INSTITUIÇÃO DE ENSINO SUPERIOR \\ DOI: http://dx.doi.org/10.5007/1983-4535.2015v8n2p28}

Tabela 5 Questões da Variável Gestão

\begin{tabular}{lcccc}
\hline \multicolumn{1}{c}{ ITEM } & \multicolumn{3}{c}{$\mathbf{\%}$} \\
\cline { 2 - 5 } & $\mathbf{N}$ & $\mathbf{E}$ & $\mathbf{F}$ & $\mathbf{S}$ \\
\hline A jornada de trabalho praticada é adequada. & - & 6,06 & 37,88 & 56,06 \\
A utilização da mão-de-obra é otimizada. & 1,52 & 16,67 & 39,39 & 42,42 \\
O retrabalho acontece. & 15,15 & 66,67 & 13,64 & 4,55 \\
Há integração entre os setores. & - & 15,15 & 45,45 & 39,39 \\
As pessoas são realocadas para setores carentes. & 21,21 & 39,39 & 25,76 & 13,64 \\
Os esforços e, ou recursos são otimizados. & - & 9,09 & 50,00 & 40,91 \\
Os setores são unificados de acordo com as atividades fins. & 1,52 & 6,06 & 40,91 & 51,52 \\
O quadro de pessoal é bem distribuído. & 4,55 & 18,18 & 46,97 & 30,30 \\
\hline \multicolumn{1}{c}{ Total } & $\mathbf{5 , 4 9}$ & $\mathbf{2 2 , 1 6}$ & $\mathbf{3 7 , 5 0}$ & $\mathbf{3 4 , 8 5}$ \\
\hline \multicolumn{2}{c}{} \\
\hline
\end{tabular}

$\mathrm{N}=$ Nunca $\mathrm{E}=$ Eventualmente $\mathrm{F}=$ Frequentemente; e $\mathrm{S}=$ Sempre

Fonte: Dados da pesquisa.

\subsection{IDADE}

No que se refere à idade, nenhum dirigente considerou a faixa etária do servidor totalmente inadequada para o desempenho de suas funções, conforme Tabela 6. Entretanto, de acordo com os dados do órgão responsável pela Gestão de Pessoas, 46,19\% dos servidores docentes e técnico-administrativos da UFV se encontram entre 50 e 60 anos e a idade média dos servidores técnico-administrativos é de 50 anos.

Como demonstrado anteriormente (Tabela 1), com as limitações de contratações de pessoal no setor público federal, formou-se uma massa de trabalhadores envelhecida e com menor condição física para o desenvolvimento de atividades, principalmente nas áreas de campo e obras.

A idade média do servidor público federal civil ativo do Ministério da Educação em 2008, segundo dados do Boletim Estatístico de Pessoal do Ministério do Planejamento, era de 46 anos para homem e 44 para mulher,

Lucena (1991) considera que as informações sobre faixas de idade oferecem subsídios importantes para o planejamento de recursos humanos no que se refere a planos de sucessão, de carreira e de desenvolvimento. Numa organização onde é grande a concentração de pessoal idoso, deve-se verificar as expectativas de aposentadorias e que cargos essas pessoas ocupam.

Tabela 6 Questão da Variável Idade

\begin{tabular}{|lcccc}
\hline & \multicolumn{3}{c}{$\%$} \\
\cline { 2 - 5 } & \multicolumn{1}{c}{$\mathbf{~}$} & $\mathbf{F}$ & $\mathbf{S}$ \\
\hline $\begin{array}{l}\text { ITEM } \\
\text { A faixa etária do pessoal é adequada ao desenvolvimento das } \\
\text { atividades. }\end{array}$ & - & $\mathbf{2 8 , 7 9}$ & $\mathbf{4 0 , 9 1}$ & $\mathbf{3 0 , 3 0}$ \\
\hline
\end{tabular}

$\mathrm{N}=$ Nunca; $\mathrm{E}=$ Eventualmente $\mathrm{F}=$ Frequentemente; e $\mathrm{S}=$ Sempre

Fonte: Dados da pesquisa 


\subsection{MOTIVAÇÃO}

$\mathrm{Na}$ opinião de $96,97 \%$ dos dirigentes, o quadro de pessoal é, frequentemente ou sempre, composto de servidores assíduos, pontuais; e 93,94\% apresentam disposição e comprometimento para com o trabalho, conforme Tabela 7.

Tabela 7 Questão da Variável Motivação

\begin{tabular}{|lcccc|}
\hline \multicolumn{1}{c}{ ITEM } & \multicolumn{3}{c}{$\mathbf{\%}$} \\
\hline & $\mathbf{N}$ & $\mathbf{E}$ & $\mathbf{F}$ & $\mathbf{S}$ \\
\hline Os servidores são assíduos. & - & 3,03 & 57,58 & 39,39 \\
Os servidores são pontuais. & - & 3,03 & 66,67 & 30,30 \\
Os servidores demonstram disposição para o trabalho. & - & 6,06 & 63,64 & 30,30 \\
Os servidores mostram-se comprometidos com o serviço. & - & 6,06 & 63,64 & 30,30 \\
\hline \multicolumn{1}{c}{ Total } & - & $\mathbf{4 , 5 5}$ & $\mathbf{6 2 , 8 8}$ & $\mathbf{3 2 , 5 8}$ \\
\hline
\end{tabular}

$\mathrm{N}=$ Nunca; $\mathrm{E}=$ Eventualmente; $\mathrm{F}=$ Frequentemente; e $\mathrm{S}=$ Sempre.

Fonte: Dados da pesquisa

Conhecer a visão do gestor quanto à motivação do quadro de pessoal é um importante instrumento de gestão, pois, segundo Herzberg, 1968 apud Lacombe, 2005, o que mais contribui para que as pessoas produzam são os fatores motivacionais. Enfatiza que a motivação das pessoas para trabalhar envolve sentimento de realização, de crescimento e de reconhecimento profissional manifestado por meio do exercício das tarefas e das atividades e afetam diretamente a produtividade.

\subsection{SAÚDE OCUPACIONAL}

Em relação à saúde ocupacional, verifica-se, conforme Tabela 8 , que $81,82 \%$ dos dirigentes consideram que, frequentemente ou sempre, os servidores apresentam adequação da saúde ocupacional para o desempenho de suas atividades.

Tabela 8 Questão da Variável Idade

\begin{tabular}{|lcccc|}
\hline & \multicolumn{3}{c}{$\%$} & $\mathbf{E}$ \\
\cline { 2 - 5 } ITEM & $\mathbf{N}$ & $\mathbf{E}$ & $\mathbf{S}$ \\
\hline $\begin{array}{l}\text { A saúde ocupacional do pessoal é adequada ao } \\
\text { desenvolvimento das atividades. }\end{array}$ & $\mathbf{1 , 5 2}$ & $\mathbf{1 6 , 6 7}$ & $\mathbf{5 1 , 5 2}$ & $\mathbf{3 0 , 3 0}$ \\
\hline
\end{tabular}

$\mathrm{N}=$ Nunca; $\mathrm{E}=$ Eventualmente; $\mathrm{F}=$ Frequentemente; e $\mathrm{S}=$ Sempre.

Fonte: Dados da pesquisa

A temática da saúde ocupacional do quadro de pessoal foi considerada no estudo, uma vez que as pressões de trabalho têm por alvo principal o corpo dos trabalhadores onde elas 


\section{ASPECTOS IMPACTANTES NO DIMENSIONAMENTO DA FORÇA DE TRABALHO EM UMA \\ INSTITUIÇÃO DE ENSINO SUPERIOR \\ DOI: http://dx.doi.org/10.5007/1983-4535.2015v8n2p28}

podem ocasionar desgaste, envelhecimento e doenças somáticas (DEJOURS et al., 1995 apud FRANÇA, 2007).

O fator saúde afeta a qualidade do trabalho, o comprometimento e a produtividade. Tem sido cada vez mais reconhecido como um elemento importante dentro das organizações, tanto pelos funcionários quanto pelos dirigentes. No serviço público também tem sido objeto de ações e medidas conforme se pode verificar através do Decreto $n^{\circ}$ 5.961/2006 (BRASIL, 2006B), que instituiu no âmbito do Sistema de Pessoal Civil da Administração Federal (SIPEC), o Sistema Integrado de Saúde Ocupacional do Servidor Público Federal (SISOSP), com a finalidade de uniformizar procedimentos administrativo-sanitários na área de gestão de recursos humanos e promover a saúde ocupacional do servidor.

\subsection{VOLUME DE TRABALHO}

Quando consultado a respeito do volume anual de trabalho dos servidores, aproximadamente $73 \%$ (Tabela 9) dos dirigentes consideraram-no, frequentemente ou sempre, uniforme. No entanto, ressalta-se que a ocorrência de sazonalidade nas atividades de algumas unidades pode levar o dirigente a reivindicar aumento no quantitativo de pessoal sem considerar a possibilidade de ações alternativas como rodízio, mutirão etc., para suprir essa necessidade temporária, podendo resultar em ociosidade.

Tabela 9 Questão da Variável Volume de Trabalho

\begin{tabular}{|lcccc|}
\hline & & & \% \\
\cline { 2 - 4 } & \multicolumn{1}{c}{ ITEM } & $\mathbf{N}$ & $\mathbf{F}$ & $\mathrm{S}$ \\
\hline $\begin{array}{l}\text { Os trabalhos rotineiros se dão em volume uniforme durante o } \\
\text { ano. }\end{array}$ & $\mathbf{4 , 5 5}$ & $\mathbf{2 2 , 7 3}$ & $\mathbf{5 7 , 5 8}$ & $\mathbf{1 5 , 1 5}$ \\
\hline
\end{tabular}

$\mathrm{N}=$ Nunca; $\mathrm{E}=$ Eventualmente; $\mathrm{F}=$ Frequentemente; e $\mathrm{S}=$ Sempre.

Fonte: Dados da pesquisa

Percebe-se, na Figura 2, maior concentração positiva de respostas nas variáveis relacionadas à ampliação, capacitação, gestão de pessoal, idade, motivação, saúde ocupacional e volume de trabalho, com exceção apenas para a variável redução da força de trabalho. Assim, pode-se inferir que todos os aspectos apontados neste estudo como importantes fatores que interferem no dimensionamento da força de trabalho, na visão dos dirigentes, estão sendo geridos de maneira satisfatória na UFV, e que a necessidade de ampliação de pessoal pode estar relacionada apenas à reposição e ampliação do quadro em virtude de novas demandas. 


\section{ASPECTOS IMPACTANTES NO DIMENSIONAMENTO DA FORÇA DE TRABALHO EM UMA \\ INSTITUIÇÃO DE ENSINO SUPERIOR \\ DOI: http://dx.doi.org/10.5007/1983-4535.2015v8n2p28}

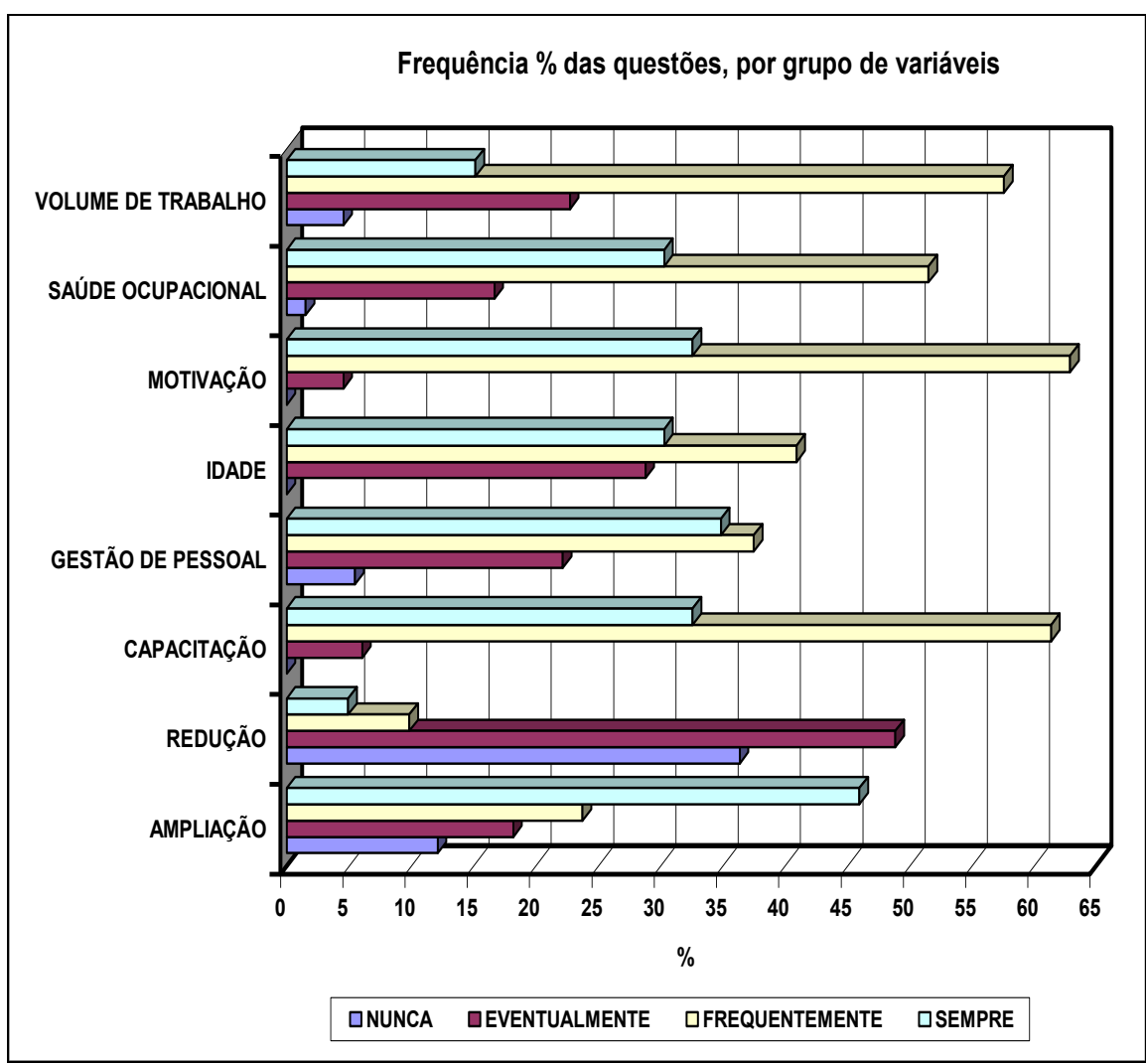

Figura 2 Consulta Global da UFV com os percentuais de cada avaliação no conjunto de grupos. Fonte: Dados da pesquisa

\section{CONCLUSÕES}

Com o objetivo de investigar variáveis que interferem no dimensionamento da força de trabalho da UFV, tentou-se identificar na percepção dos dirigentes como se encontra o gerenciamento de pessoas nos aspectos relacionados a capacitação, motivação, idade, saúde ocupacional, otimização de recursos, para, por fim, verificar se há necessidade de ampliação da força de trabalho em termos de planejamento de curto e de longo prazos.

Os resultados demonstram que, na percepção dos dirigentes, para atender às demandas e ao processo de expansão institucional em curso, é necessário ampliar o quadro de pessoal, uma vez que há bom gerenciamento de pessoas.

Entretanto, pela análise dos dados secundários verifica-se que em aspectos como capacitação e idade, as respostas dos dirigentes não condizem com as políticas efetivamente adotadas pela UFV.

Ressalta-se que a pesquisa foi efetuada no início do processo de expansão das instituições federais de ensino, o que pode ter repercutido na administração dos recursos 
humanos, uma vez que, para cumprir os objetivos pactuados na expansão, os dirigentes se sentem pressionados a demandar mais recursos, sejam eles materiais, financeiros ou humanos.

Além disso, após longo período de redução do quadro de pessoal, em razão das medidas governamentais impostas, e o momento atual da expansão institucional, a tendência natural é que o dirigente manifeste que a força de trabalho em seu poder é insuficiente para alcançar os objetivos institucionais.

Uma questão importante a ser aqui considerada é: Se o diagnóstico fosse feito num momento de estabilidade institucional os dirigentes teriam uma postura mais exigente e qualitativa em relação à avaliação dos variáveis pesquisadas? Pois não se pode negar que a inovação tecnológica, a modernização dos processos de trabalho, a motivação, a capacitação e o gerenciamento eficazes da força de trabalho levam a Instituição à otimização, sem que haja necessidade de ampliar o quadro de pessoal.

A inexistência de instrumento baseado em critérios para definição de alocação de vagas coloca o dirigente numa posição gerencial fragilizada, não permitindo ser preciso na indicação de necessidade de pessoal. Esta situação também pode ocorrer em virtude da falta de investimento na formação gerencial dos dirigentes, embora no momento da pesquisa a maioria dos participantes contava com experiência de, no mínimo, quatro anos na função.

É importante salientar que o processo de planejamento de recursos humanos deve ser uma prática permanente e contínua na Instituição, pois tanto a Instituição quanto as pessoas mudam e, com isso, as suas necessidades também. A avaliação e o planejamento da força de trabalho devem trazer à discussão as variáveis que impactam na gestão de pessoas.

Sugere-se para novas pesquisas a ampliação do número de atores sociais pesquisados, contemplando os servidores subordinados aos dirigentes, propiciando maior reflexão e também maior investigação das variáveis relativas à gestão de pessoas, além da possibilidade de confrontação das opiniões.

Por fim, esta investigação mostrou que tão importante quanto dimensionar a força de trabalho necessária ao atendimento das necessidades institucionais é identificar e conhecer o quanto a organização dispõe de pessoal para suprir as suas necessidades. Também é importante identificar e conhecer: os indicadores para equacionar essas necessidades a curto, médio e longo prazos; o nível de capacitação do quadro de pessoal; as necessidades de adequação da política para gestão de pessoas; os processos essenciais da instituição; a 
complexidade tecnológica; os processos de mudança; e o ambiente. Portanto, conclui-se que se trata de um campo fértil para pesquisas com benefícios práticos e teóricos.

\section{REFERÊNCIAS}

BRASIL. Decreto 5.825, de 29 de junho de 2006. Estabelece as diretrizes para elaboração do Plano de Desenvolvimento dos Integrantes do Plano de Carreira dos Cargos TécnicoAdministrativos em Educação, instituído pela Lei no 11.091, de 12 de janeiro de 2005. Diário Oficial (da República Federativa do Brasil), Brasília, DF. 30 jun. 2006.

. Decreto 5.961/2006, de 13 de novembro de 2006. Institui o Sistema Integrado de Saúde Ocupacional do Servidor Público Federal - SISOSP. Diário Oficial (da República Federativa do Brasil), Brasília, DF. 14 nov. 2006 b.

. Lei ${ }^{0} 11.091$, de 12 de janeiro de 2005. Dispõe sobre a estruturação do Plano de Carreira dos Cargos Técnico-Administrativos em Educação, no âmbito das Instituições Federais de Ensino vinculadas ao Ministério da Educação, e dá outras providências. Diário Oficial (da República Federativa do Brasil), Brasília, DF. 13 jan. 2005.

. Lei $\mathrm{n}^{\circ} 8.112$, de 11 de dezembro de 1990. Dispõe sobre o regime jurídico dos servidores públicos civis da União, das autarquias e das fundações públicas federais. Diário Oficial (da República Federativa do Brasil), Brasília, DF. 12 dez. 1990.

. Ministério do Planejamento, Orçamento e Gestão/Secretaria de Recursos Humanos. Boletim Estatístico de Pessoal no 09 de 1997 e n 158 de Dez 2008.

CHIAVENATO, I. Recursos humanos: o capital humano das organizações. São Paulo: Atlas, 2006.

DESSLER, G. Administração de Recursos Humanos. 2 ed. Tradução Cecília Leão Oderich. São Paulo: Prentice Hall, 2003.

DUTRA, J.S. Gestão de Pessoas. Modelo, Processos, Tendências e Perspectivas. São Paulo: Atlas, 2002.

FERREIRA, M. R. de L.; GOMES, F.P.; ARAÚJO, R.M. Gestão de Pessoas no Setor Público: um estudo dos níveis de conflito a partir da visão interacionista. Encontro de Administração Pública e Governança. Salvador. BA, 12-14 nov.2008.

FRANÇA, A.C. Práticas de Recursos Humanos - PRH: confeito, ferramentas e procedimentos. São Paulo: Atlas, 2007.

GAIDZINSKI, R.R. O dimensionamento de pessoal de enfermagem em instituições hospitalares. (Ttese doutorado). São Paulo: Escola de Enfermagem da USP; 1998.

YIN, K. R. Estudo de caso: planejamento e métodos. 2 ed. Porto Alegre: Bookman, 2002. 
LACOMBE, B.M.B; CHU, R.A. Políticas e Práticas de Gestão de Pessoas: As abordagem estratégica e institucional. Revista de Administração de Empresa. v. 48, n. 1, JanMar/2008.

LACOMBE, F.J.M. Recursos Humanos: princípios e tendências. São Paulo: Saraiva, 2005.

LUCENA, M.D.S. Planejamento de Recursos Humanos. São Paulo: Atlas, 1991.

MARCONI, Nelson. Gestão de Recursos Humanos nas organizações sociais. In: IX Congresso Internacional Del CLAD sobre la Reforma Del Estado y de la Administracion Púlica. Madrid. Espanha, 2 -5- Nov. 2004.

MORGAN, D. L. Focus group as qualitative research. Sage university paper series in: Qualitative research methods. London: Sage Publications, 1997.

NOGUEIRA, R. P. Novas Tendências Internacionais da Força de Trabalho do Setor Público O Brasil comparado com Outros Países. ObservaRH. UNB. Brasília. 2005.

PACHECO, R. S. Política de recursos humanos para a reforma gerencial: realizações do período 1995-2002. RSP - Revista do Serviço Público. Ano 53, n. 4, Out-Dez 2002.

SABIONI, Gustavo Soares; NUNES, Vívian Kelly Andaki; REIS, Cisne Zélia Teixeira (Orgs.). Relatório Anual de Atividades da UFV - 2008. UFV, PPO, Viçosa, MG, 2009. $103 p$.

SABIONI, Gustavo Soares; NUNES, Vívian Kelly Andaki; REIS, Cisne Zélia Teixeira (Orgs.). Relatório Anual de Atividades da UFV - 2007. UFV, PPO,Viçosa, MG, 2008. $101 \mathrm{p}$.

SABIONI, Gustavo Soares; GUIO, Leanice Maria Peruzzo; REIS, Cisne Zélia Teixeira (Orgs.). Relatório Anual de Atividades da UFV - 2003. UFV, PPO,Viçosa, MG, 2004. $106 \mathrm{p}$.

VERGARA, S. C. Projetos e Relatórios de Pesquisa em Administração. 7 ed. São Paulo: Atlas, 2006.

UFV. UNIVERSIDADE FEDERAL DE VIÇOSA. Relatório de Gestão do ano 2008. Viçosa-MG. 2009. 